\title{
Diet sampling by wild Norway rats offered several unfamiliar foods
}

\author{
MATTHEW BECK, CHRISTINE L. HITCHCOCK, and BENNETT G. GALEF, JR. \\ McMaster University, Hamilton, Ontario, Canada
}

\begin{abstract}
The present experiment was undertaken to examine directly the diet sampling behavior of wild Norway rats (Rattus norvegicus) faced with a choice among familiar and unfamiliar foods. Firstgeneration, laboratory-reared wild Norway rats ate from four food cups. Three of the food cups were in unfamiliar locations and contained unfamiliar foods. The remaining food cup was in a familiar location and contained a familiar food. Subjects in a control group were offered the familiar food in all four locations. We found (1) that subjects in experimental and control conditions took equal amounts of time to first visit food cups in unfamiliar locations, (2) that subjects in the experimental condition (those with access to unfamiliar foods) ate at unfamiliar locations at a slower rate than did subjects in the control condition (those with access only to familiar food), (3) that subjects in the experimental condition were no more likely than subjects in the control condition to eat at one unfamiliar location at a time, and (4) that following a bout of eating at an unfamiliar food cup, subjects in the experimental condition waited no longer than subjects in the control condition before eating from a different unfamiliar food cup. We interpreted these data as indicating that although wild Norway rats are hesitant to eat unfamiliar foods, once they begin to eat such foods, they do not sample among them so as to facilitate identification of any toxin present.
\end{abstract}

To survive, wild Norway rats, like other omnivores selecting foods in complex environments, must both eat a nutritionally adequate mix of foods and avoid ingesting lethal quantities of toxic substances. Selection of nutritionally adequate diets and avoidance of ingestion of toxins can be somewhat contradictory objectives. Acquisition of all necessary micronutrients may require that an omnivore increase the number of different foods it eats. Increasing dietary breadth may, in turn, increase an omnivore's probability of ingesting a toxin. In consequence, omnivores could benefit from using a sampling strategy that held ingestion of toxins to a minimum while permitting evaluation of the postingestional consequences of any foods eaten. Such a sampling strategy would seem to require, at the least, that rats and other omnivores behave differently when ingesting unfamiliar, potentially dangerous foods than when ingesting familiar, safe foods. Rats should eat small initial meals of unfamiliar, potentially dangerous foods. They should eat meals containing only one unfamiliar food at a time. They should, as rats clearly do, learn more rapidly to associate negative aftereffects of ingestion with unfamiliar foods than with

\footnotetext{
This research was supported by grants to B.G.G., Jr., from the Natural Sciences and Engineering Research Council of Canada and the McMaster University Research Board. During the conduct of this study, M.B. held an Ontario Graduate Scholarship and C.L.H., a Natural Sciences and Engineering Research Council of Canada Summer Studentship. We thank Elaine Whiskin for her technical assistance and Harvey Weingarten for his help with earlier drafts of the manuscript. C.L.H. is now at the Department of Psychology, University of Toronto. Requests for reprints should be sent to B. G. Galef, Jr., Department of Psychology, McMaster University, Hamilton, ON L8S 4K1, Canada.
}

familiar ones (Kalat \& Rozin, 1973; Revusky \& Bedarf, 1967).

If an omnivore gorged on each unfamiliar food it encountered, the ability to learn rapidly to associate the taste of an unfamiliar food with toxicosis would sometimes be of little use; at least some unfamiliar, toxic foods would prove fatal following a first, large meal. If an omnivore were to sample several unfamiliar foods at a time, it would have difficulty avoiding subsequent ingestion of any unfamiliar, toxic foods that it had eaten. Strong aversions might develop to the most salient (Kalat \& Rozin, 1970) or, perhaps, the most recently eaten unfamiliar food, but salience or recency of ingestion are not necessarily reliable guides to toxicity. Cautious ingestion of one unfamiliar food at a time would seem to maximize an individual's chances both of surviving initial encounters with unfamiliar foods and of associating a poisonous food with its consequences.

Rozin and Kalat (1971) first proposed that "the rat's feeding pattern maximizes the possibility of associating each diet with its appropriate consequences, since meals tend to be isolated in time and consist of a single food" (p. 465). Similar assertions have been made subsequently by others: "Rats in fact do tend to sample new foods, which are the ones that would naturally be most likely to be poisonous, and wait long enough between meals for poisoning to take effect"' (Shettleworth, 1984, p. 26). "[Rats] exhibit ... feeding patterns most likely to facilitate the accurate identification of foods which cause illness, frequently eating only one food during a feeding bout"' (Zahorik \& Houpt, 1981, p. 293). Although there is consensus in the literature concerning the behavior of 
rats sampling among unfamiliar foods, the evidence supporting this consensus is not very convincing.

A large number of studies have described the "neophobia" of wild rats, that is, their hesitancy to begin eating an unfamiliar food (Barnett, 1958; Cowan, 1977; Galef, 1970; Mitchell, 1976; Rozin, 1968; Rzòska, 1953; Shorten, 1954). However, evidence of reluctance to begin eating unfamiliar foods does not bear directly on the issue of whether rats sample among several unfamiliar foods so as to increase their ability to associate each food with its consequences. Rozin (1969), most frequently cited as providing evidence that rats sample unfamiliar foods one at a time so as to facilitate poison identification, demonstrated that, over a period of several days, 4 of 10 thiamine-deficient domesticated rats sampled among three unfamiliar foods and came to eat only the thiamine-rich alternative. These data do not show (and Rozin has not claimed that they show) that rats encountering several unfamiliar, potentially dangerous foods sample among them so as to facilitate identification of a toxic food should one be present. To the contrary, Rozin's (1969) data indicated that many of his domestic rat subjects ate two or three unfamiliar foods during the first $\mathbf{3 0}$ min those foods were available.

Such promiscuous sampling of unfamiliar foods is not restricted to domesticated rats. Barnett (1956) found that when four unfamiliar foods were offered to firstgeneration, laboratory-bred wild rats for the first time, "it was usual for all four foods to be eaten within the first feeding period" (p. 30). Absence of discrete sampling of unfamiliar foods by wild rats is clear from each of several of Barnett's descriptions of the behavior of a wild rat facing a choice among novel foods. For example,

Eating begins with 15 minutes' intermittent consumption of flour with some sniffing of the other foods. Liver is eaten for $2 \mathrm{~min}$, then still holding a piece of liver, leans into sugar box and eats sugar, leaves piece of liver at back of cage, returns to liver tin and eats liver for $1 \mathrm{~min}$. Eats sugar for $5 \mathrm{~min}$. Restless interval of $4 \mathrm{~min}$ followed by picking up bits of liver and dropping them; eats wheat for $1 \mathrm{~min}$, then sniffles in the wheat tin. Followed by $42 \mathrm{~min}$ of restlessness with some sampling of wheat, flour and sugar, before settling to sleep (Barnett, 1956, p. 32)

It is difficult to see how a rat that suffered toxicosis after sampling among four unfamiliar foods in this way would know which food to avoid in the future.

One reason that the literature on diet sampling by wild rats is less complete than one might wish is that, until relatively recently, it has been difficult to acquire detailed descriptions of the diet sampling patterns of free-feeding rats. Without a microcomputer to monitor behavior continuously at a number of different feeding stations, measurement of food sampling by free-feeding rats required full-time observation by an experimenter (see, e.g., Thompson, 1948). The present experiment was undertaken to exploit the power of the microcomputer to determine whether rats do, in fact, sample unfamiliar foods in the adaptive fashion that has been suggested in the literature.

\section{METHOD}

\section{Subjects}

The subjects were 24 6- to 10-week-old, first-generation, laboratory-bred, wild Norway rats (Rattus norvegicus) selected from six litters born in the McMaster vivarium. Each litter was born to a different breeding colony, consisting of 2 male and 2 female wild rats trapped in a livestock bam in West Flamborough, Ontario. Each breeding colony was housed in a $1 \times 1 \mathrm{~m}$ enclosure and maintained on Purina Laboratory Rodent Chow and water, available ad lib in a temperature- and humidity-controlled room on a 12:12 light:dark cycle. Two pups from each litter were assigned to the control condition, and 2 were assigned to the experimental condition described below in Procedure. Data on diet sampling was not obtained from 4 subjects ( 3 in the experimental condition and 1 in the control condition) because they failed to eat at unfamiliar locations prior to the end of the experiment.

\section{Apparatus}

Throughout the 4 days of the experiment, each subject was individually housed and tested in one of four $60 \times 60 \times 30 \mathrm{~cm}$ enclosures (see Figure 1) constructed of transparent Plexiglas (walls, feeding shelves, feeding drawers, and floor), galvanized sheet metal (removable litter tray and protective covers for feeding shelves), and hardware cloth (lid). Each enclosure contained four cylindrical food cups ( $5 \mathrm{~cm}$ diam $\times 5 \mathrm{~cm}$ deep), each recessed in a separate $7.5 \times 30 \mathrm{~cm}$ Plexiglas drawer concealed beneath one of the 7.5-cmwide $\times 5-\mathrm{cm}$-deep feeding shelves running along two walls of each enclosure.

Drawers containing food cups were constructed so that, if opened by the experimenter (as illustrated in Figure 1, Cup 2), the lid on each drawer blocked an opening in a feeding shelf. If a drawer was pushed closed, a 5-cm-diam hole in the lid of the drawer provided access to a food cup through a corresponding opening in a feeding shelf. Thus, subjects could eat from a food cup when the drawer containing it was closed, but not when it was open.

Photocells and associated lights were mounted in the floor of each feeding shelf on either side of each of the 5-cm-diam openings leading to each of the four food cups. These photocells were each connected via amplifier and interface to a Tandy 1200 microcomputer, programmed to record the time of occlusion and of unblocking of each photocell. Each photocell was examined by the computer for change in level of illumination every $1.04 \mathrm{sec}$.

The Plexiglas floor of each enclosure was covered with a removable $60 \times 60 \mathrm{~cm}$ sheet metal litter pan, carpeted with a thin layer of wood-chip bedding. Resting on the bedding in each enclosure was an opaque $15 \times 7.5 \times 7.5 \mathrm{~cm}$ glass bottle that provided a place of refuge for each subject.

The four enclosures were located in a single temperature- and humidity-controlled room continuously illuminated by a single 40 $\mathrm{W}$, red light bulb hanging $1 \mathrm{~m}$ above the level of the boxes and equidistant from their centers.

\section{Procedure}

At 2 p.m. on Day 1 of the experiment, four littermates were trapped from a breeding colony and introduced individually into the four test enclosures. At the time of introduction of each subject into its enclosure, powdered Purina Laboratory Rodent Chow was available in Food Cup 1. The remaining three feeding drawers in each enclosure were open and empty. The subjects were left undisturbed for $48 \mathrm{~h}$ to become habituated to the apparatus.

At the end of the 48-h habituation period (at 2 p.m. on Day 3 of the experiment), all four food cups in the test enclosures of sub- 


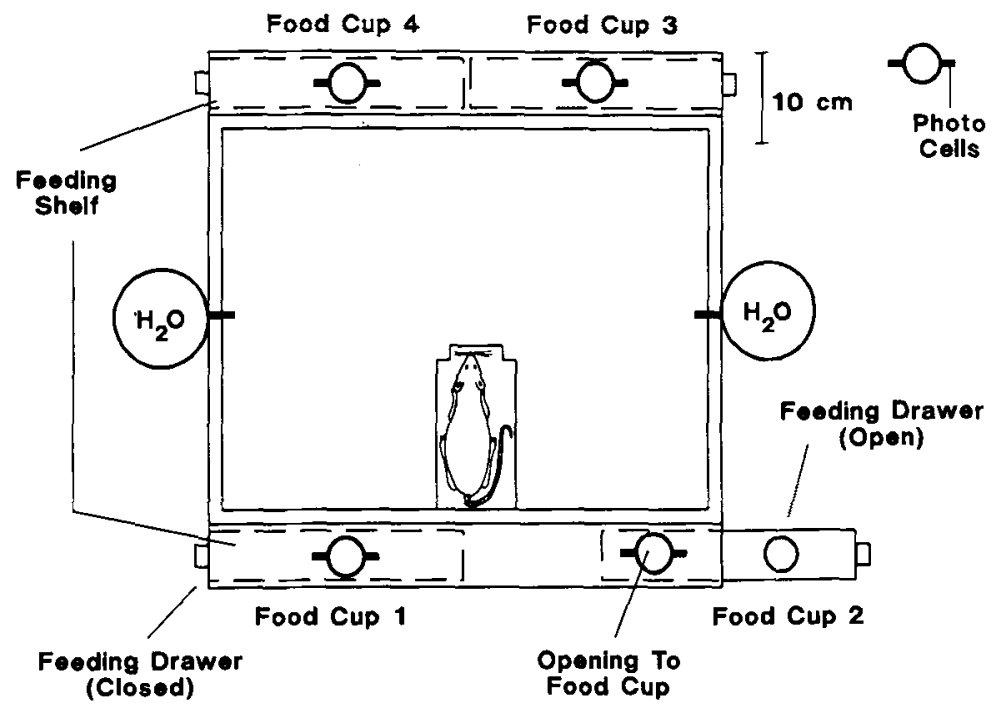

Figure 1. Overhead schematic of an experimental enclosure showing feeding drawers in open and closed positions.

jects assigned to the control group were filled with weighed samples of powdered Purina Laboratory Rodent Chow and closed. At the same time, the food cups of each subject assigned to the experimental group were filled with weighed samples of four different foods and closed. Food Cup 1 was refilled with powdered Purina Laboratory Rodent Chow; Food Cup 2, with a cinnamon-flavored diet (Teklad Normal Protein Test Diet mixed with $1 \% \mathrm{wt} / \mathrm{wt}$ McCormick's Fancy Ground Cinnamon); Food Cup 3, with cocoa-flavored diet (Teklad Normal Protein Test Diet mixed with $2 \%$ wt/wt Hershey's cocoa); and Food Cup 4 with marjoram-flavored diet (Teklad Normal Protein Test Diet mixed with 2.3\% McCormick's marjoram). Normal Protein Test Diet (Teklad Test Diets, Madison, WI) is a nutritionally adequate diet composed largely of casein (26\%), corn starch $(60 \%)$, and vegetable and cod liver oils (10\%). It is preferred by wild rats to powdered Purina Laboratory Rodent Chow in a ratio of 3 or 4 to 1 in a simple choice test.

Each subject was left undisturbed from 2 p.m. on Day 3 of the experiment until 2 p.m. on Day 4, when the food cups were weighed, refilled, and reweighed. Following this 30-min interruption, each subject was again left undisturbed for a final $231 / 2 \mathrm{~h}$. The experiment was terminated at 2 p.m. on Day 5.

\section{Data Analysis}

The computer was programmed to store the times when each of 4 rats caused a change in the illumination level of the photocell monitoring each of its four food cups. In the course of $24 \mathrm{~h}$, a single rat caused as many as $\mathbf{5 7 4}$ such events. To proceed with analysis of the data, it was necessary to reduce this wealth of data points to a more manageable number of feeding bouts and meals.

Feeding bouts. We defined any two occlusions of the same photocell that occurred within $15 \mathrm{sec}$ of one another and without an intervening occlusion of another photocell as part of the same feeding bout. The length of a feeding bout was defined as the number of seconds a photocell was occluded within that bout.

Meals. We treated any two feeding bouts occurring within $15 \mathrm{~min}$ of one another as part of the same meal (Kissileff, 1970). In determining intermeal intervals, we ignored feeding bouts less than $2 \mathrm{sec}$ in duration, since observation of subjects on closed-circuit television had indicated that no ingestion occurred during such very brief contacts with food. The duration of a meal was defined as the number of seconds photocells were occluded during that meal.

Any criteria used for data reduction are, obviously, arbitrary. If we had wished to establish the absolute duration of feeding bouts or absolute length of intermeal intervals, the criteria we employed would have had important effects on our findings. However, because we were interested in differences in the feeding patterns of wild rats faced with familiar and unfamiliar foods, the absolute values of feeding bouts and intermeal intervals were not critical.

\section{RESULTS}

\section{Correlations Between Time Spent Eating and Amount of Food Eaten}

Because our apparatus measured the number of seconds each subject spent with its head in each food cup rather than the number of grams of food each subject ate from each food cup, our index of ingestion was indirect rather than direct. We did, however, know both how much food was eaten from each food cup by each subject and how long each subject spent with its head in each food cup. It was therefore possible to calculate linear regressions of amount of time spent eating on amount of food eaten.

As can be seen in Table 1, correlations between amount eaten and time spent eating were very good on Day 3 of the experiment, the critical day. Consequently, conclusions concerning amount of food eaten, but based on measurement of time spent eating from the various food cups on Day 3, are informative with respect to diet sampling.

The main source of error in the correlation is suggested by the observed positive intercept of all four regression

Table 1

Pearson Product Moment Correlations Between Time Spent Eating from a Food Cup (sec) and Amount Eaten from that Food Cup (g)

\begin{tabular}{clccccc}
\hline Day & Group & $r_{x y}$ & $p$ & $r_{x y}^{2}$ & Slope & $y$ Intercept \\
\hline 3 & Expt'l & .92 & $<.001$ & .85 & .0029 & .58 \\
4 & Control & .93 & $<.001$ & .86 & .0029 & .67 \\
3 & Expt'l & .89 & $<.001$ & .79 & .0030 & .87 \\
4 & Control & .68 & $<.001$ & .46 & .0022 & 1.69 \\
\hline
\end{tabular}


lines when one would expect the best-fitting lines to pass through the ordinate. The positive $y$ intercepts suggest, and observation of spillage around food cups indicated, that subjects occasionally used their paws to scoop food from the food cups and later ate the spillage. Food cups designed to prevent such behavior would have improved the validity of our dependent variable.

\section{Evidence of "Neophobia" in Eating Unfamiliar Foods}

If wild Norway rats sample unfamiliar foods cautiously, then one would expect subjects in our control group (which had their familiar food in Food Cups 2, 3, and 4) to eat from Food Cups 2, 3, and 4 at a higher rate than did the subjects in our experimental group (which had three unfamiliar foods in Food Cups 2, 3, and 4). The left-hand panel of Figure 2 shows the mean number of minutes required for subjects in experimental and control groups to eat for $n$ sec from whichever of Food Cups 2,3 , or 4 they first ate from for $n$ sec. The right-hand panel of Figure 2 shows the mean number of minutes required for subjects in experimental and control conditions to eat for $n$ sec from whichever of Food Cups 2, 3, or 4 they next ate from for $n$ sec.

As is evident from inspection of Figure 2, although initial exploration of first and second food cups by subjects in experimental and control groups occurred at roughly the same time (both $U s>34$, n.s.), subjects in the control group ate from unfamiliar food cups at a greater rate than did subjects in the experimental group (see Figure 2 for $U$ and $p$ values). These data are consistent with the generally held view that wild rats eat unfamiliar foods more slowly or cautiously than they eat familiar foods. The failure of 3 of 12 subjects in the experimental group and of only 1 of 12 in the control group to eat from Cups 2,3 , or 4 during the 2 days of the experiment similarly provides evidence of the hesitancy of wild rats to ingest unfamiliar foods.

\section{Evidence of Discrete Sampling of Unfamiliar Foods}

If wild rats sample among unfamiliar foods so as to permit ready identification of a toxic food should one be present, then (1) meals that contain an unfamiliar food should be concentrated on a single unfamiliar food, and (2) rats should tend to wait longer between meals containing unfamiliar foods than between meals not containing unfamiliar foods.

Concentration of meals on a single unfamiliar food. To determine whether meals containing unfamiliar foods tended to be concentrated on one unfamiliar food, we examined each meal eaten by each subject to determine whether the subject ate from Food Cups 2, 3, or 4 during that meal. If a subject ate from Food Cups 2, 3, or 4 during a meal, we then determined (1) which unfamiliar food cup it ate most from during that meal (the modal unfamiliar food cup) and (2) the percentage of total intake from Food Cups 2, 3, and 4 eaten from the modal unfamiliar food cup.

If rats tend to eat one unfamiliar food at a time, then meals containing an unfamiliar food should be more concentrated on a single unfamiliar food cup than similar
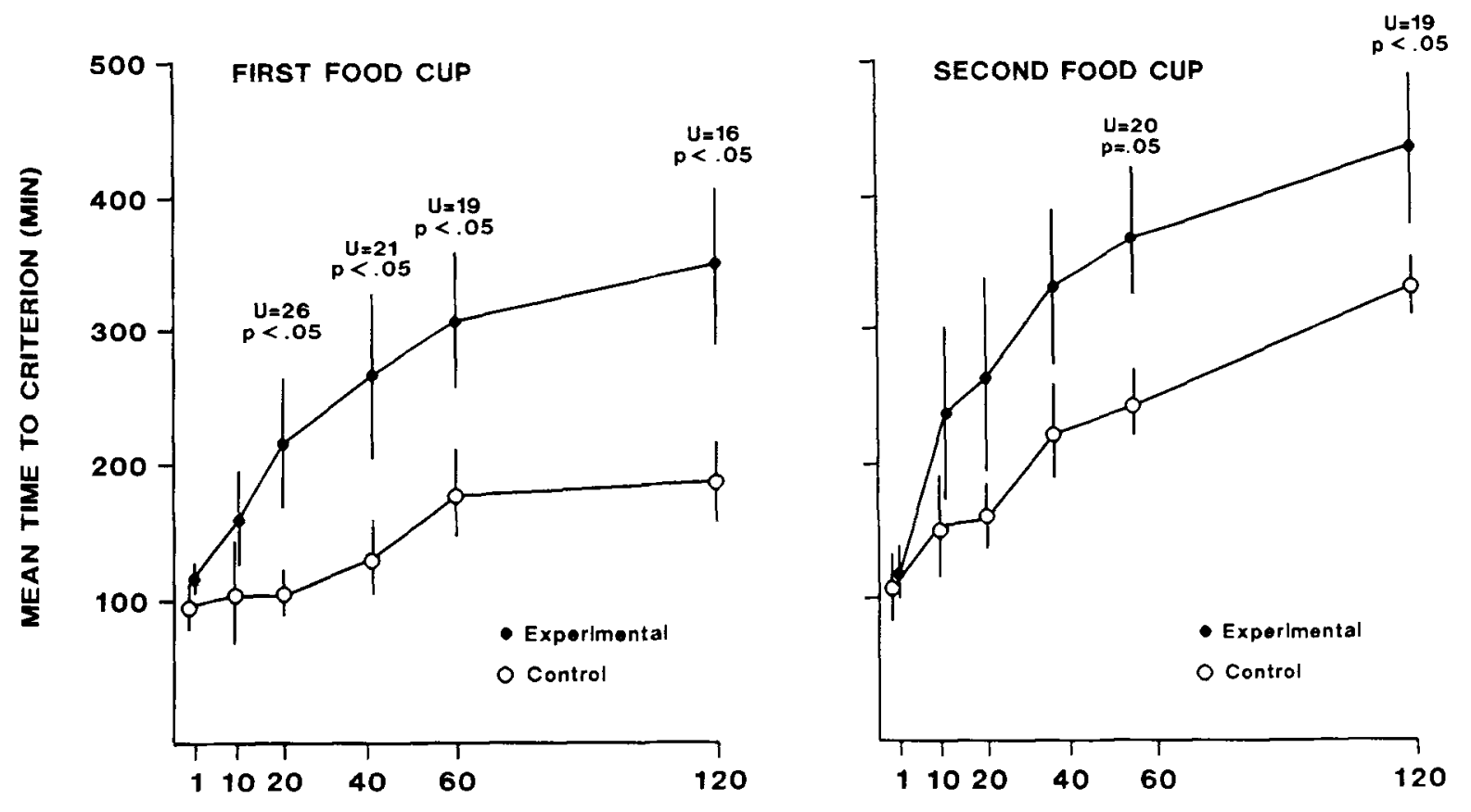

CUMULATIVE TIME EATING (SEC)

Figure 2. Mean number of minutes required for subjects in experimental and control groups to eat from an unfamiliar food cup for a criterial number of seconds. Left-hand panel: First unfamiliar food cup exploited for criterial number of seconds by each subject. Right-hand panel: Second unfamiliar food cup exploited for criterial number of seconds by each subject. Flags $=>1$ SEM. 
meals eaten by subjects in the control group (that consisted only of familiar food). Figure 3 shows the mean percentage eaten from the modal unfamiliar food cup by subjects in the experimental and control groups for the first eight meals each subject ate that contained a feeding bout at Food Cups 2, 3, or 4. As can be seen in Figure 3, there was no tendency for subjects in the experimental group (those eating unfamiliar foods) relative to subjects in the control group (those eating only familiar food) to concentrate their feeding on a single unfamiliar food cup. Thus, our data were not consistent with the view that wild rats tend to eat one unfamiliar food at a time.

Temporal patterning of meals of unfamiliar foods. The question of whether rats wait longer after eating a meal containing an unfamiliar food before eating a second such meal than they do between meals of a familiar food is less easily addressed. The problem is that rats are very variable, both in the sizes of the meals they eat and in the patterning of those meals. Indeed, it is difficult to know exactly how rats sampling unfamiliar foods should behave to maximize feeding efficiency while evaluating the postingestive effects of each unfamiliar food they eat. Much depends on the nature of the toxins with which rats have to contend. If the toxic dose of a poisonous food were small and the onset of toxicosis were long-delayed, the adaptive strategy to be employed in sampling unfamiliar foods would be different from that useful with poisonous foods having large toxic doses and rapid onset of toxicosis (Freeland \& Janzen, 1974).

In preparing each of the four panels of Figure 4, we assumed that rats needed to ingest differing amounts of a food to become ill and that the effects of ingesting an illness-producing sample were delayed for varying amounts of time. For example, the upper left-hand panel of Figure 4 analyzes the behavior of experimental and

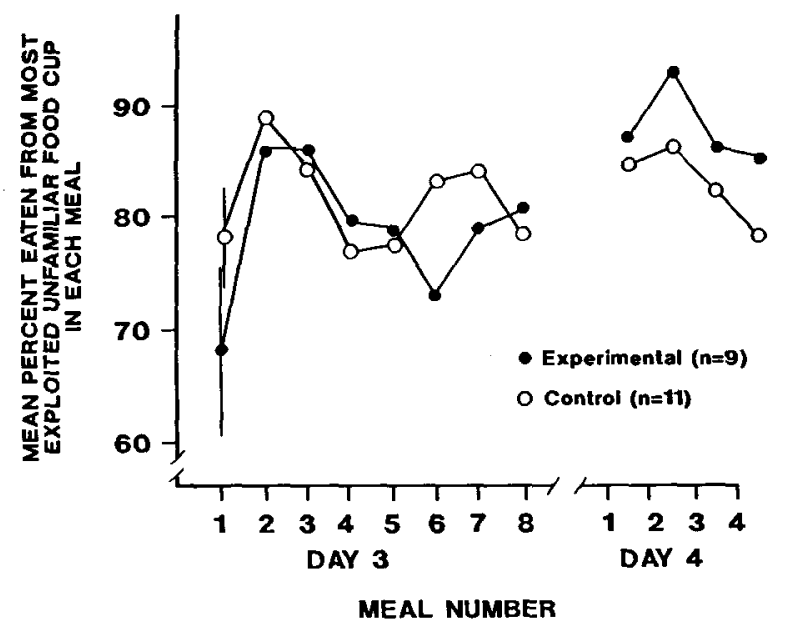

Figure 3. Mean length of intervals between successive meals of subjects in experimental and control conditions on Days 3 and 4 of the experiment. See Data Analysis section of Method for definition of a meal. control subjects on the assumption that $20 \mathrm{sec}$ of eating an unfamiliar food provides a toxic dose and that the onset of symptoms of illness occurs with latencies of from 5 to $120 \mathrm{~min}$. This panel shows the percentage of subjects in experimental and control conditions eating for $20 \mathrm{sec}$ from a second unfamiliar food cup (Food Cups 2, 3 , or 4) within 5-120 min of eating for $20 \mathrm{sec}$ from a first unfamiliar food cup. Thus, the upper left-hand panel of Figure 4 shows, for instance, that $70 \%$ of the experimental subjects failed to wait for $30 \mathrm{~min}$ after eating their first unfamiliar food for $20 \mathrm{sec}$ before eating their second unfamiliar food for $20 \mathrm{sec}$. Similarly, the upper right-hand panel of Figure 4 shows the percentage of subjects in experimental and control conditions eating for $\mathbf{4 0} \mathrm{sec}$ from one unfamiliar food cup within 5-120 min of eating for $40 \mathrm{sec}$ from another unfamiliar food cup. Together, the four graphs in Figure 4 show the percentage of subjects that would have eaten illness-producing samples of two different unfamiliar foods before the onset of toxicosis (1) as a function of the time to onset of toxicosis following ingestion of a toxic food, and (2) assuming various different size threshold toxic doses of unfamiliar foods (upper-left, $20 \mathrm{sec}$; upper-right, $40 \mathrm{sec}$; lower-left, $60 \mathrm{sec}$; lower-right, $120 \mathrm{sec}$ ).

Each subject had access to three unfamiliar food cups. Each subject in the experimental group could sample three unfamiliar foods, and each subject in the control group could eat at three unfamiliar locations. In consequence, each subject provided two indices of sampling, one involving the first and second unfamiliar food cups or foods it exploited and the other involving the second and third unfamiliar food cups or foods it exploited. In Figure 4, the letters A and B refer, respectively, to the first and second pairs of unfamiliar food cups exploited by subjects.

In making comparisons among panels, two things should be kept in mind: (1) The unfamiliar food cup eaten from first for $20 \mathrm{sec}$ is not necessarily the same food cup eaten from first for 40,60 , or $120 \mathrm{sec}$, and (2) during the experiment, 2 subjects did not sample from all three unfamiliar food cups for $120 \mathrm{sec}$. These two factors can produce what appear to be inconsistencies across panels.

The first point to be made about Figure 4 is that the absolute values in the four panels are of little interest. The absolute values reflect the effects of variables such as the handling times of foods, travel times between food cups, length of time subjects were habituated to their enclosures, and so forth. What is of interest is comparison of the sampling patterns exhibited by subjects in the control condition (eating a familiar food at unfamiliar locations) and subjects in the experimental condition (eating unfamiliar foods at unfamiliar locations). Subjects in the experimental group should, on the prevailing model, be less likely than subjects in the control group to eat a criterial amount from two different unfamiliar food cups within any period of time. This prediction was clearly not confirmed by the data. Subjects in the experimental condition were, if any- 

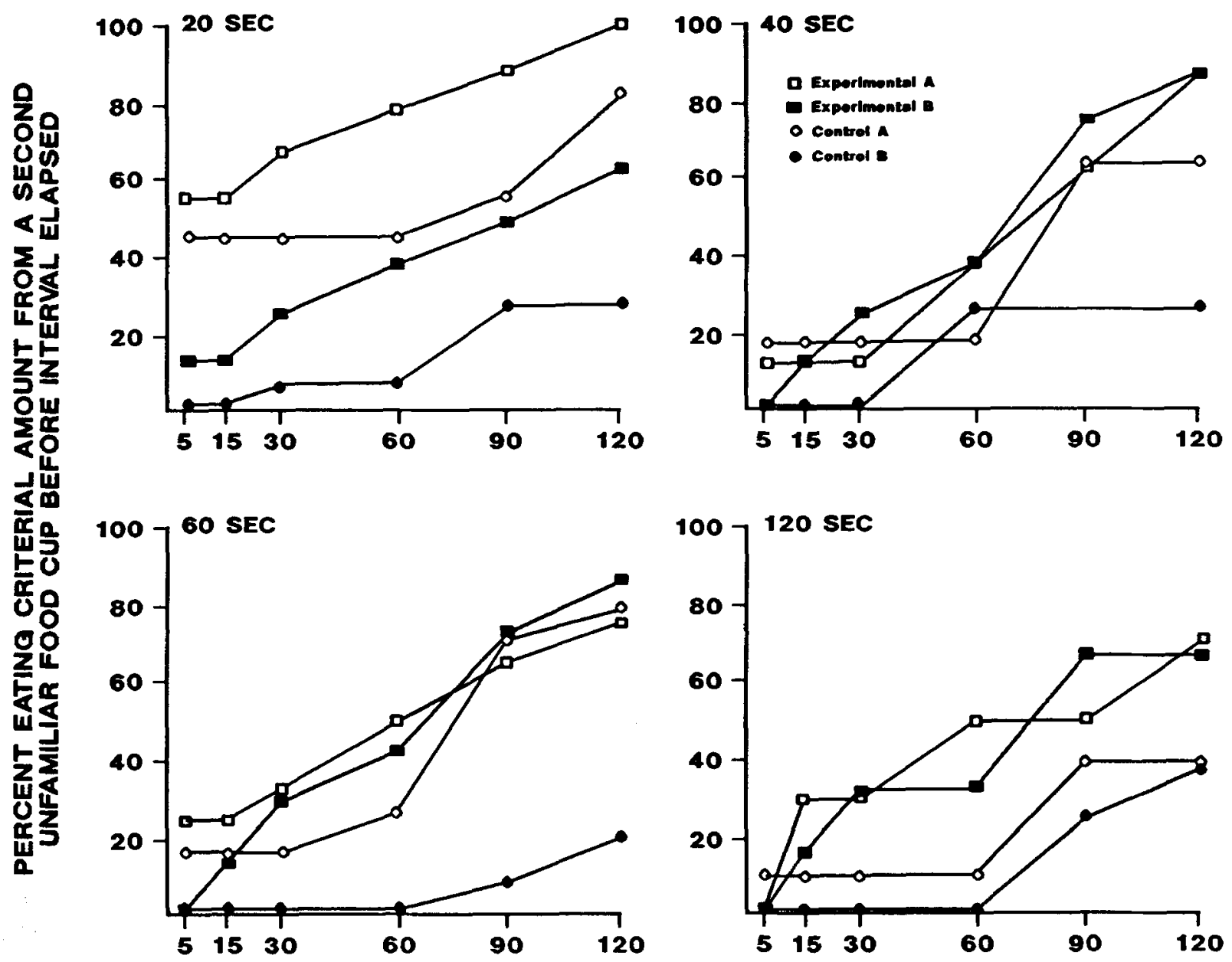

INTERVAL (MIN)

Figure 4. Percentage of control and experimental subjects eating for $n_{i}$ sec from a second unfamiliar food cup within $n_{j}$ min of having eaten for $\boldsymbol{n}_{i}$ sec from a first unfamiliar food cup. See Results section for explanation of groups.

thing, more likely than subjects in the control condition to eat a criterial amount from two unfamiliar food cups within any given period of time.

\section{DISCUSSION}

The results of the present experiment provide confirmation of one hypothesis concerning the feeding behavior of wild rats; our wild rats, like everyone else's, did eat unfamiliar foods at a slower rate than they ate familiar foods. The results of the present experiment failed to confirm two other hypotheses concerning the feeding behavior of wild rats: (1) Our experimental subjects did not eat one unfamiliar food at a time, and (2) they did not pause for an extended period between meals of different unfamiliar foods. In sum, our wild rat did not sample unfamiliar foods so as to facilitate independent evaluation of the consequences of eating each food.

The hypothesis that rats eat only one novel food at a time and wait after eating an unfamiliar food to evaluate its postingestional effects carries the implication that rats have evolved a specific strategy for coping with environments containing a number of unfamiliar, potentially toxic foods. The results of the present study suggest that wild rats do not, in fact, sample among scattered unfamiliar foods very differently than they sample among similarly distributed portions of familiar food. Consequently, our data offer no support for the hypothesis that wild Norway rats have evolved a strategy of discrete sampling among unfamiliar foods.

Of course, failure to observe a pattern of behavior is never definitive. Perhaps our measures were insensitive. Perhaps the conditions of our experiment were inappropriate to elicit discrete sampling of unfamiliar foods. Still, subjects in our study, like those whose behavior has been described by Barnett (1956) and Rozin (1969) in rather different situations, failed, at first encounter, to sample one at a time among several unfamiliar foods. Consistent failure to find such discrete sampling of unfamiliar foods suggests that discrete sampling is not a species-typical 
strategy used by Norway rats to facilitate the identification of any toxin present in an array of unfamiliar foods.

Discrete sampling of unfamiliar foods is not necessarily the optimal strategy available either to wild rats or to other free-living omnivores when faced with an array of unfamiliar foods. If, for example, rats evolved in environments in which toxic foods were rarely encountered, they might have benefited from a strategy of eating a little of each of a number of different unfamiliar foods in rapid succession. Discrete sampling among unfamiliar foods might be exhibited only by those rats that experienced illness after engaging in such an initial bout of promiscuous feeding.

The important implication of the present data, when considered together with those of Barnett (1956) and Rozin (1969), is that there is no basis for asserting that rats have evolved a strategy of eating one unfamiliar food at a time. Without evidence of such an adaptation, it is not parsimonious to assert its existence (Williams, 1966). Rats seem to sample among unfamiliar foods at unfamiliar feeding sites as much as they sample among several portions of a familiar food in the same locations.

\section{REFERENCES}

BARNETT, S. A. (1956). Behaviour components in the feeding of wild and laboratory rats. Behaviour, 9, 24-42.

BARNETT, S. A. (1958). Experiments on 'neophobia' in wild and laboratory rats. British Journal of Psychology, 49, 195-201.

Cowan, P. E. (1977). Neophobia and neophilia: New object and new place reactions of three Rattus species. Joumal of Comparative \& Physiological Psychology, 91, 63-71.

Freeland, W. J., \&ANZEN, D. H. (1974). Strategies in herbivory by mammals: The role of secondary plant compounds. American Naturalist, 108, 269-289.

GALEF, B. G., JR. (1970). Aggression and timidity: Responses to novelty in feral Norway rats. Journal of Comparative \& Physiological Psychology, 70, 370-381.
Kalat, J. W., \& Rozin, P. (1970). "Salience": A factor which can override temporal contiguity in taste-aversion learning. Journal of Comparative \& Physiological Psychology, 71, 192-197.

Kalat, J. W., \& Rozin, P. (1973). Learned safety as a mechanism in long-delay taste-aversion learning in rats. Journal of Comparative \& Physiological Psychology, 83, 198-207.

KISSILEFF, H. (1970). Free-feeding in normal and "recovered lateral" rats monitored by pellet detecting eatometer. Physiology \& Behavior, $5,163-173$.

MitcheLL, D. (1976). Experiments on neophobia in wild and laboratory rats: A reevaluation. Journal of Comparative \& Physiological Psychology, 90, 190-197.

Revusky, S. H., \& BEDARF, E. W. (1967). Association of illness with prior ingestion of novel foods. Science, 155, 219-220.

RozIN, P. (1968). Specific aversions and neophobia resulting from vitamin deficiency or poisoning in half-wild and domestic rats. Journal of Comparative \& Physiological Psychology, 66, 82-88.

RozIN, P. (1969). Adaptive food sampling patterns in vitamin deficient rats. Joumal of Comparative \& Physiological Psychology, 69, 126-132.

Rozin, P., \& Kalat, J. W. (1971). Specific hungers and poison avoidance as adaptive specializations of learning. Psychological Review, 78, 459-486.

RzòskA, J. (1953). Bait shyness, a study in rat behaviour. British Journal of Animal Behaviour, 1, 128-135.

SHETTLEWORTH, S. J. (1984). Function and mechanism in learning. In M. Zeiler \& P. Harzem (Eds.), Biological factors in learning (pp. 163). New York: Wiley.

ShORTEN, M. (1954). The reaction of the brown rat toward changes in its environment. In D. Chitty (Ed.), Control of rats and mice (Vol. 2, pp. 307-334). Oxford: Clarendon Press.

THOMPSON, H. V. (1948). Studies of the behaviour of the common brown rat (Rattus norvegicus, Berkenhout). Bulletin of Animal Behaviour, 6, 26-40.

WILLIAMS, G. C. (1966). Adaptation and natural selection. Princeton, NJ: Princeton University Press.

ZAHORIK, D. M., \& HouPT, K. A. (1981). Species differences in feeding strategies, food hazards, and the ability to leam food aversions. In A. C. Kamil \& T. D. Sargent (Eds.), Foraging behavior (pp. 289310). New York: Garland Press.

(Manuscript received August 24, 1987; revision accepted for publication December 21, 1987.) 